\title{
Carcinoma Breast, Late Presentation-A Big Concern
}

\author{
MN ASLAM A K ANSARI A SIDDIQUE MIMRAN \\ Department of Surgery, King Edward Medical University/Mayo Hospital, Lahore \\ Correspondence to Dr. Muhammad Nadeem Aslam, Assistant Professor Surgery
}

\begin{abstract}
Objectives: To study the different presentation patterns and their treatment options in advanced carcinoma breast.
Design: A retrospective observational study

Settings: North Surgical Ward, Mayo Hospital|, Lahore, from November 2004 to April 2006.

Patients and Methods: In total, 156 patients with carcinoma breast, confirmed histopathologically by biopsy and /or FNAC, were included in this study. Information was gathered from them using a structured questionnaire.

Results: The peak incidence of carcinoma breast was found to be between 31- 45 years of age. Majority of these patients presented in stage II (22\%). Maximum number of patients presented in stage III (56\%). However stage IV disease was also found in $12 \%$ of patients' population. But stage I disease was least commonly presented disease (10\%). And infiltrative ductal carcinoma was the most prevalent $(94 \%)$ histologic type. Modified radical mastectomy was the surgical treatment adopted in about $72 \%$ of cases with adjuvant or neoadjuvant chemotherapy.

Conclusion: Advance stage breast lump with local or locoregional spread remained the commonest mode of presentation of carcinoma breast. And infiltrative ductal carcinoma is the commonest histologic variant. Majority of the of patients with carcinoma breast in Pakistan still present in advanced stages where almost no cosmetically more acceptable surgical procedure can be carried out.
\end{abstract}

Key words: $\mathrm{CA}$ breast, late presentation, modified radical mastecvtomy

The new millennium still finds breașt cancer as a leading cause of cancer related deaths in females all over the world. The incidence of carcinoma breast in Pakistan stands $24.4 \%$, thus making it the commonest malignancy amongst Pakistani females. Similarly, carcinoma breast is also the leading cause of deaths in females in the country. The majority of the patients present in the late stage of the disease and even the multimodal treatment cannot improve overall morbidity and mortality in these patients ${ }^{1}$.

In outpatients of almost all of our hospitals, most of the patients of carcinoma breast present lately in the disease course. This includes the entire spectrum of the disease like locally advanced disease, locoregionally advanced disease and the disease with visceral and skeletal metastases ${ }^{1}$. This late presentation is multifactorial both on the part of the patients as well as of the clinician who sees and assesses the patient first of all ${ }^{13}$.

The stage of the disease at the time of presentation is the single most important predictor of the over all morbidity and mortality of the patient. Likewise the stage at the commencement of the treatment would be the most important determinant of final cosmetic results in a particular patient. Early presentation offers a wider range of surgical options both on technical as well as cosmetic grounds. ${ }^{5}$ In the advance stage of the disease the management options are limited and in certain settings no surgical treatment can be undertaken because of the advance stage of the malignancy. And left over options are chemoradiation. In other instances, the only surgical option is debulking of the disease where not even the tumour is removed completely ${ }^{5,14}$.

There have always been growing concerns about cosmessis among patients of carcinoma breast . But they generally fail to understand the direct relationship of post surgical cosmetic results with disease stage at the time of first ever presentation to a clinician. Therefore this late presentation of breast malignancy is a major concern and needs to be rectified ${ }^{12,14}$.

\section{Materials and methods:}

This study was planned as a retrospective observational study. The study was carried out in the Department of Surgery, Mayo Hospital, Lahore during November 2004 to April 2006. One hundred and fifty six patients presented with carcinoma breast were included in this study. All cases presented between 25 and 80 years of age and the peak incidence was seen in 31-45 years of age.

A thorough history and examination was obtained for each case. The findings were then subjected to statistical analysis. All the patients included in this study were having proven breast carcinoma either on FNAC or histopathologically.

All the patients were worked up through a set pattern of history, examination and investigations. This workup. took into account the features like mode of presentation, histologic diagnosis, stage of disease, other diagnostic investigations and treatments offered.

\section{Results:}

Age Distribution: Among the 156 patients, it was seen that the age varied from 25 to 80 years and the peak incidence was seen in $31-45$ years of age.

Table 1

\begin{tabular}{lll}
\hline Age(Years) & Age group & Incidence $(\%)$ \\
\hline$<30$ & Young & 6 \\
$31-45$ & Middle aged & 58 \\
$46-60$ & Perimenopausal & 28 \\
$>60$ & Old & 8 \\
\hline
\end{tabular}


Mode of presentation: Painless lump breast was the commonest mode of presentation found in $76 \%$ patients while painful breast lump/ mastalgia was found only in $24 \%$ of patients. Both were associated with nipple retraction in $4 \%$ and metastatic presentation in $10 \%$.

Tumor site: $60 \%$ of the patients had the tumor in the upper outer quadrant of breast followed by upper inner quadrant(16\%), lower outer quadrant(12\%) and lower inner quadrant $(8 \%)$ The central area was the least common site with only $4 \%$

Table 2:

\begin{tabular}{ll}
\hline Site & Incidence $(\%)$ \\
\hline Upper outer quadrant & 60 \\
Upper inner quadrant & 16 \\
Lower outer quadrant & 12 \\
Lower inner quadrant & 8 \\
Central area & 4 \\
\hline
\end{tabular}

Stage at time of presentation: Out of 156 patients, $56 \%$ presented with stage III. This was followed by stage II in $22 \%$ of patients. Stage I and stage IV were less prevalent with $10 \%$ and $12 \%$ respectively. Nodal involvement was seen in $30 \%$ of patients

Stage IV has less survival ratio as they may not reach hospital or they go directly to Oncology.

Figure 1

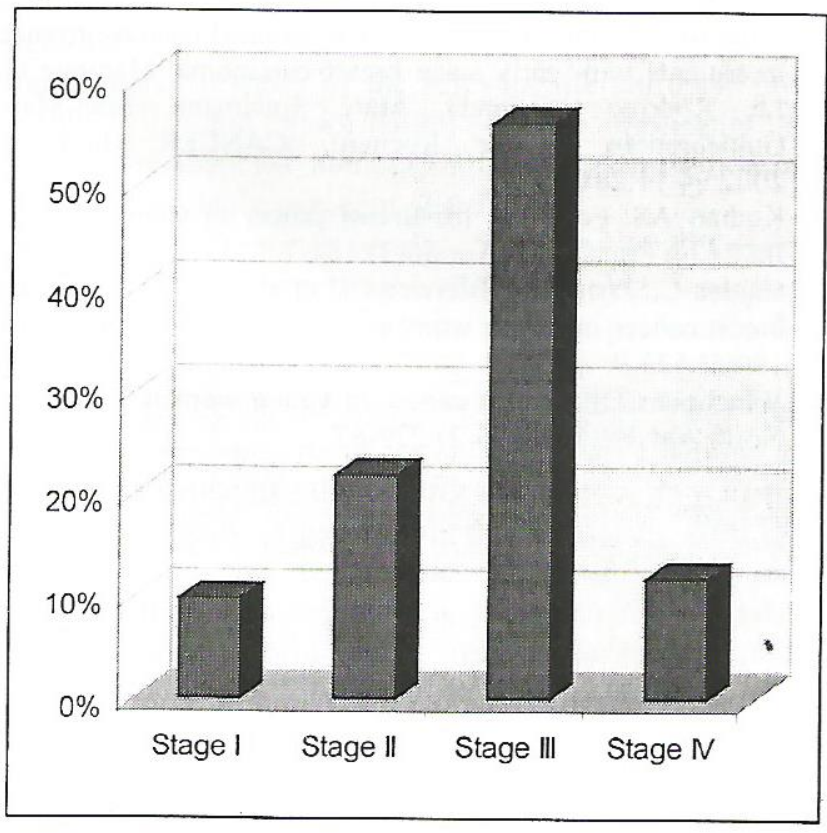

Histologic types:Infiltrative ductal carcinoma was the commonest phenotype with $94 \%$ while the lobular and the mixed variety were very rare with $2 \%$ each.

Diagnostic Tool: $72 \%$ of the patients were diagnosed by surgical biopsy while the remaining $28 \%$ were diagnosed with FNAC.
Treatment modalities: $72 \%$ patients underwent modified radical mastectomy, $2 \%$ radical mastectomy, $4 \%$ wide local excision, $8 \%$ simple mastectomy, $4 \%$ neoadjuvant chemotherapy and $2 \%$ lumpectomy. $2 \%$ of the patients expired before any treatment, $2 \%$ refused to give consent for surgery and $4 \%$ left against medical advice(LAMA) Recurrence: Only 10\% patients had recurrent disease.

\section{Discussion:}

Accurate statistical data regarding incidence of carcinoma breast is unavailable. However, unofficial sources identify it as the commonest malignant disease of the female gender $^{1,2,3}$.

It was observed that most of the patients presented with late stage carcinoma. Collectively $68 \%$ patients presented with stage III and IV. On the other hand, 32\% presented with stage I and II. It is believed that there is often a delay in the diagnosis of breast cancer in young women ${ }^{12-14}$.

Breast cancer treatment is no longer a decision to be made by the surgeon single handedly. There is an established role of oncologist, medical and radiation oncologist, radiologist and pathologist.

Carcinoma breast can be treated through various modalities of treatment. Multimodal management is especially required for locally advanced disease or the one with visceral or skeletal metastasis ${ }^{4,7}$.

The extent of surgical procedure is directly dependent on the stage of the disease. Earlier the presentation, simpler would be the surgical procedures. The operations for carcinoma breast range from wide local excision, or lumpectomy, to modified radical mastectomy ${ }^{11}$.

Simple procedures are associated with cosmetically more acceptable results than more extensive surgical procedure. Better cosmetic results are brought about by a recommended protocol of management termed as Breast Conservation Therapy. Several other factors are also considered while planning for breast conservation treatment ${ }^{12}$.

Non availability or contraindications of radiotherapy, tumor involving reteroareolar area, previous irradiation and multicentric disease are important considerations for Breast Conservation Therapy. Moreover, breast carcinoma treatment is not at all a choice for locally advanced disease or the tumor with regional or distant spread.

Despite of wide spread surgeries performed for breast carcinoma; there is a need among clinicians and practitioners to have awareness about early presentation of carcinoma breast. Because it is not only the awareness of patients but also of the clinicians which helps in the commencement of the management. The delay in the diagnosis of carcinoma breast is partly attributed to the failure of clinicians ${ }^{12}$.

The range of surgical procedures for carcinoma breast includes MRM, simple mastectomy, debulking mastectomy, radical mastectomy and wide local excision. 
Unless the number of women undergoing ablative !urgeries for carcinoma breast is brought down, the combined efforts of breast conservation/reconstruction will remain a practical challenge for the new surgeons. ${ }^{14}$

First level of attention is to train the clinicians about the early presentation of the disease. The next level of attention is better service organization in the management of breast carcinoma. These patients present late because of social taboos. ignorance and lack of facility. On late presentation, cosmesis becomes difficult and more . ggressive treatment becomes very much wanted $^{13}$.

There is a need to work for awareness among the clinicians to emphasize that treatment of carcinoma breast is not confined to remove the affected breast only. Neither . $t$ is a decision to be made by the practitioner who first sees he patient. Moreover, opinion from the specialties like ncology, radiotherapy, plastic surgery must also be sought whenever required. With growing concerns about -osmetically acceptable management of carcinoma breast, It has become a must not to decide breast removal without ionsulting other specialties' doctors. ${ }^{13,14}$

Patients of developed countries present more at an early stage because of breast cancer screening programs. 3reast screening may detect clinically asymptomatic and nence, smaller, more treatable, breast cancers. Currently in UK. breast screening consists of regular two view bilateral mammography for age range 50-64 years ${ }^{2,3}$

To follow the patient up is the integral part of the treatment but, unfortunately, we lack this in our country. surgical treatment for CA breast provides a wide range of options, but due to lack of follow up, the surgeons limit inemselves just to MRM. This also holds true in this study. Furthermore, the breast reconstruction cannot be provided ue to same reason. ${ }^{1}$

\section{Conclusion:}

- Delayed presentations can be prevented by the patient ¿ducation about different grades of the disease. Also the facilities for patient' assessment should also be made more widely available.

- Data analysis in all hospitals should be updated in order to remain in touch with the late presentation and subsequent outcome after the treatment in patients of carcinoma breast.

- Frequent arrangements should be made for different breast camps for case identification.

\section{References:}

1. Rasool MI, Malik MI, Luqman M, Khalilullah. The clinicopathological study of CA breast, Pak Med Res 1987; 6:1356

2. Cuschieri A, Giles GR and Mossa AR (1985). Essential surgical practice, $3^{\text {rd }}$ edition. Oxford, ButterworthHeinemann.

3. Dixon JM (1997) ABC of Breast diseases, London, BMJ publishing group.

4. Harris JR, Hellman S, Silen, W, (eds) conservative management of breast cancer, Lippincot 1983; 77-90.

5. Shuster JD, girshovich L, Whitney TM, Hughes KS, Multidisciplinary care for patients with breast cancer, Sur Clin North AM 2000:80;508-9.

6. Larson D, Weinstein M, Goldberg I,

7. Ferguson DJ, the actual extent of mastectomy: a key to survival. Perspect Biol Med 1987;30:311-23.

8. Borst MJ, Ingold JA: metastatic patterns of invasive lobular versus invasive ductal carcinoma of breast, Surgery 1993 October. 114(4):637-41, discussion 641-2

9. Prognostic factors for death after an isolated local recurrence in patients with early stage breast carcinoma, Manique G. Le, Rodrigo Arriagada, Marc Spielmann, Jean-Marc Guinebretiere, France Rochard, CANCER; June 1, 2002;94:11;2813-20.

10. Kothari AS, Fentiman IS, Breast cancer in young women. Int J Clin Pract. 2002 Apr 56(3):184-7.

11. Gajdos C, Tartter PI, Bleiweiss IJ et al stage 0 to stage III breast cancer in young women, J Am Coll Surg 2000 May; 190(5):523-9.

12. Winchester DP, Breast cancer in young women, Surg Clin North AM 1996 Apr 76(2):279-87. 